\section{Idiopathic Recurrent Stupor, Still an Unsolved Issue}

\section{Chiara Fois ${ }^{1}$, Bastianino Murgia ${ }^{1}$, Rossella Avallone ${ }^{2}$, Raffaela Maria Murrighile ${ }^{1}$ and GianPietro Sechi ${ }^{1}$}

\begin{abstract}
We report a case of unexplained recurrent stupor for which, given the current knowledge and the lack of easily available diagnostic tools, we were unable to reach a definite diagnosis. In the patient the syndrome was associated with an ominous prognosis. Worldwide accepted and easily available proceeding method is needed to manage patients with recurrent stupor for which a toxic origin may be supposed.
\end{abstract}

\section{Keywords}

Idiopathic recurrent stupor; Endozepine stupor; Munchausen syndrome

\section{Introduction}

Since 1992 the existence of a clinical entity characterized by unexplained recurrent hypersomnolence, named Idiopathic Recurrent Stupor (IRS), and has been an interesting matter of debate [1]. Recently, Plazzi et al [2] stated that, at present, IRS has to be considered an unproven and questionable entity and it is therefore mandatory, before making this diagnosis, to perform sophisticated toxicological tests to rule out the presence of exogenous benzodiazepines (BZDs). Unfortunately these tests cannot be easily done in the setting of routine clinical practice, and in some patients with unexplained recurrent hypersomnia the diagnosis of IRS might be, mistakenly, still supposed.

\section{Case Report}

We studied a 76-year old man who was admitted to our hospital in March 2011, after having been found unconscious at home. He was partially aroused only by vigorous tactile stimuli. Glasgow Coma Scale was 6; ocular examination showed a slight bilateral miosis with preserved but torpid pupillary reflex; oculo-cephalic reflex was present. Vital parameters and brain CT scan were normal. Blood and urine samples for routine and toxicological tests were collected. An electroencephalogram (EEG) showed a widespread 13-18 Hz fast activity, prominent over the frontal regions, on an otherwise normal waking background rhythm. When vocally and manually stimulated, or during eye opening, the patient's EEG showed a paradoxical reaction with a temporary return of alpha-like frequency. Sample tests were clear from toxic substances (benzodiazepines, barbiturates,

*Corresponding author: Chiara Fois, MD, Department of Clinical and Experimental Medicine, Unit of Neurology, University of Sassari, Viale San Pietro, 1007100 Sassari, Italy, Fax: 0039079 228423; Tel: 0039079 228231; E-mail: chiarafois@hotmail.it

Received: July 23, 2015 Accepted: November 23, 2015 Published: November 28, 2015 tricyclic antidepressants, amphetamine, cocaine, alcohol, opioids, and cannabinoids). Lumbar puncture was performed and cerebrospinal fluid (CSF) chemical analysis was unremarkable. He gradually and spontaneously recovered from the stupor after three days. On day four, he was totally oriented and able to behave completely normal, although he was not able to recall anything happened during the previous event. Repeated EEGs were normal and he was discharged in healthy condition.

After the first episode he came to our emergency department six more times, nearly every two months, in the same state of stupor. Brain magnetic resonance imaging (MRI), single photon emission tomography, brain angio-MRI, cardiological check, abdominal ultrasound, interictal one-night polysomnography recording, electromyography study, immunology-wide screening and neuropsychological assessment were normal. In each occasion blood and urine tests were repeated, but failed to detect any hint of substance abuse.

For the first time, in our clinical experience, we thought to be facing a case of possible endozepine stupor, a condition characterized by episodes of stupor purportedly linked to elevated serum level of the endogenous benzodiazepine receptor ligand, endozepine-4 $[1,3,4]$. In the light of negative exam results and based on the clinical and EEG features, which totally resembled those seen in IRS, from the second episode we decided to try flumazenil. What happened was a sudden, although temporary (5-10 minutes), change in the patient's EEG, which returned to a normal alpha rhythm (Figure 1), and a prompt return of consciousness. We repeated flumazenil each time he came to our hospital in the stupor condition since it was the only effective therapy we could offer him at that time. Moreover, we decided to check the patient's blood and cerebral spinal fluid samples for the presence of endozepine-4. This was done through an experimental lab in Emilia-Romagna where a Liquid chromatography-Mass Spectrometry (LC-MS) detecting method was available, according to what suggested by Cortelli et al. [4]. Disappointingly, this exam turned out negative for endozepine. Unfortunately, the labs in our region were not able to perform a more sophisticated assessment other than traditional Gas-Chromatographic-Mass-Spectrometric (GCMS) for ruling out exogenous benzodiazepine receptor agonists. Indeed, more advanced recommended methodic such as LC-MS or High Performance Liquid chromatography-electrospray tandem mass spectrometry (HPLC-MS-MS) are mostly used for research purpose.

Although some peculiar features in the family history pointed to a possible Munchausen syndrome by proxy, no concrete evidence came out even after the patient and his family had been questioned different times. The patient became more and more frustrated for the condition which we could not explain or stop and he died after committing suicide.

\section{Discussion and Conclusions}

We want to point out the difficulty you might find while evaluating people with unexplained IRS, since, as already warned, there are a lot of toxicological substances such as triazolam, bromazepam, lorazepam as well as zopiclone, zolpidem and zalepon that are very 
Citation: Fois C, Murgia B, Avallone R, Murrighile RM, Sechi GP (2015) Case Report: Idiopathic Recurrent Stupor, Still an Unsolved Issue. J Sleep Disor: Treat Care 4:4.

\section{(-) $06 / 06 / 201112.33 .41$}

(+) Filtro PA: $0.53 \mathrm{~Hz}$ Filitro PB : $30.0 \mathrm{~Hz}$ Ampiezza EEG: 70 microVolttcm Notch si

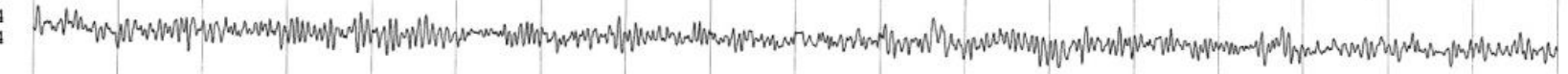

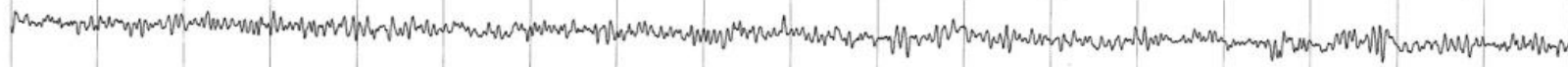

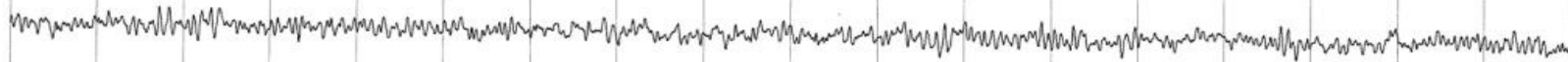

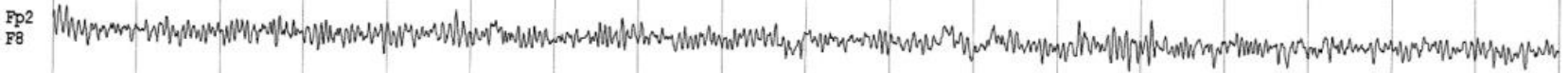

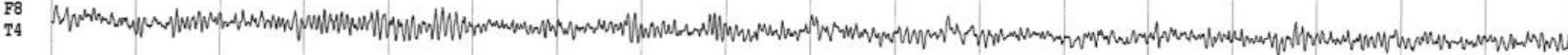

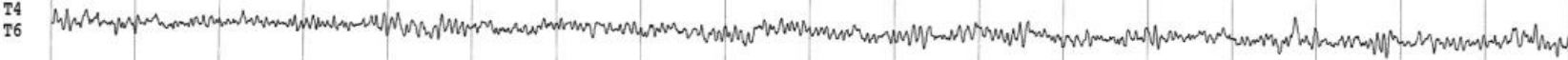

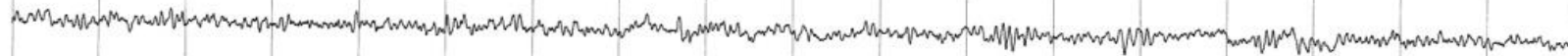

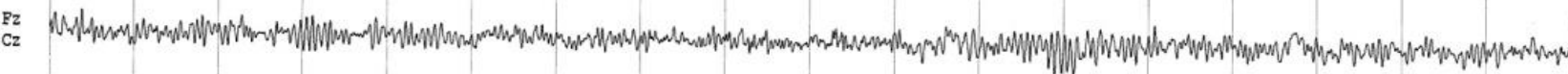

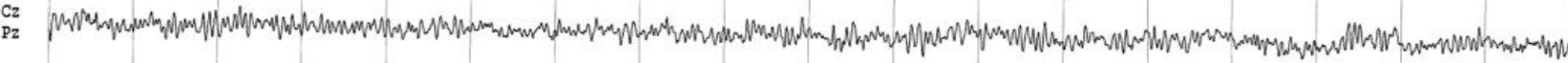

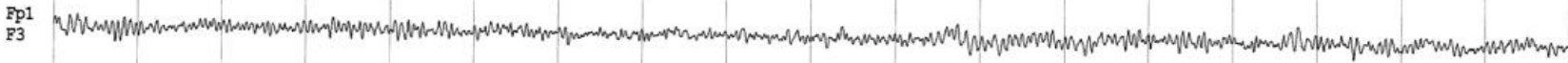

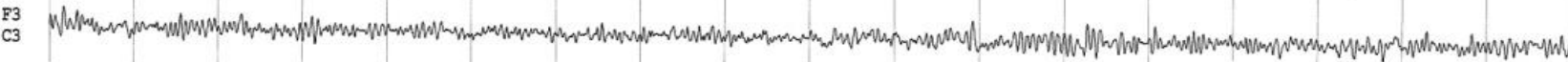

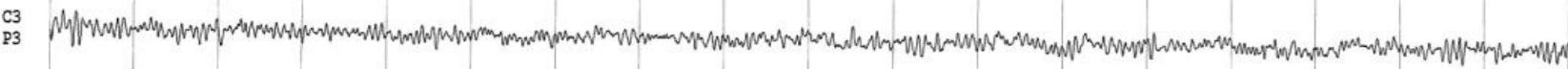

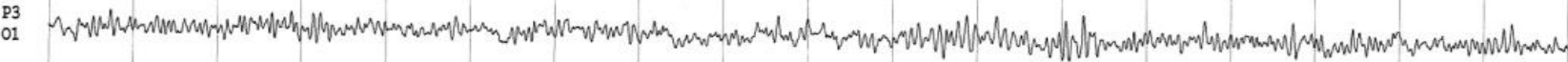

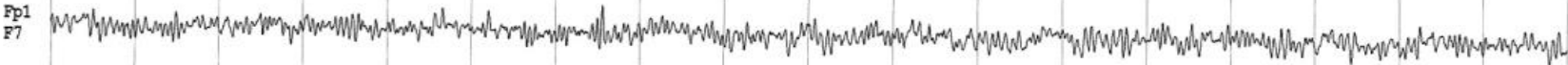

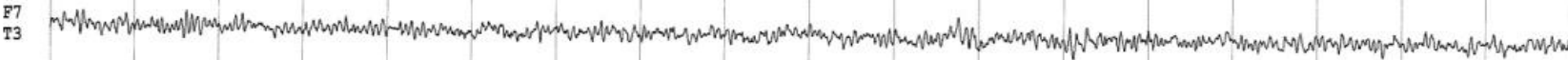

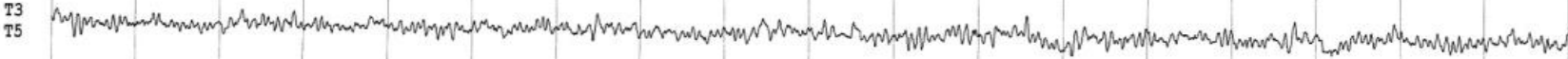

25
01

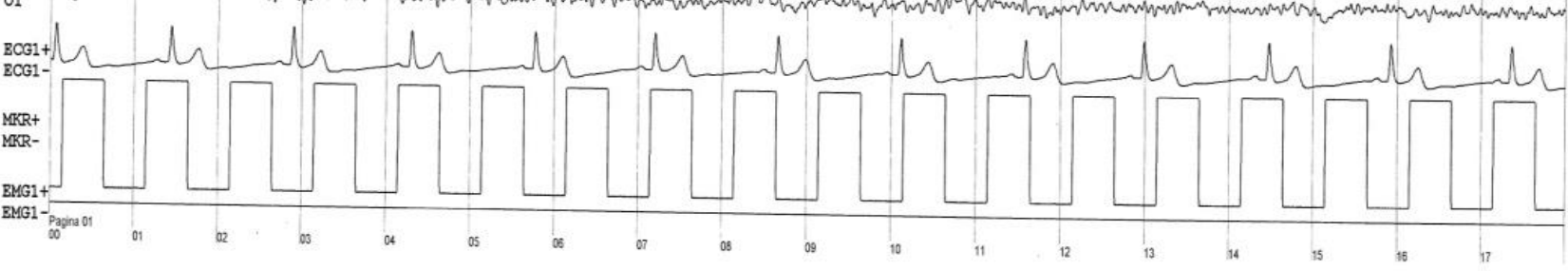


Citation: Fois C, Murgia B, Avallone R, Murrighile RM, Sechi GP (2015) Case Report: Idiopathic Recurrent Stupor, Still an Unsolved Issue. J Sleep Disor: Treat Care 4:4.

$\begin{array}{lll}(-) & 06 / 06 / 2011 & 13.18 .43 \\ (+) & \text { Filtro PA } 053\end{array}$

(+) Filtro PA: $0.53 \mathrm{~Hz}$ Filtro PB : $30.0 \mathrm{~Hz}$ Ampiezza EEG: 70 microvatucom Notch si

B

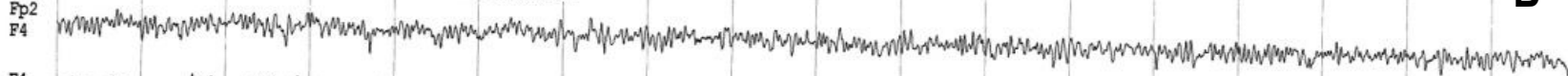

F4

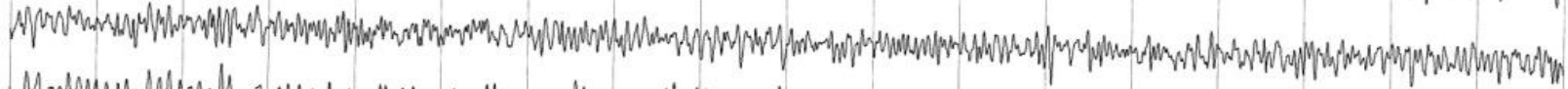

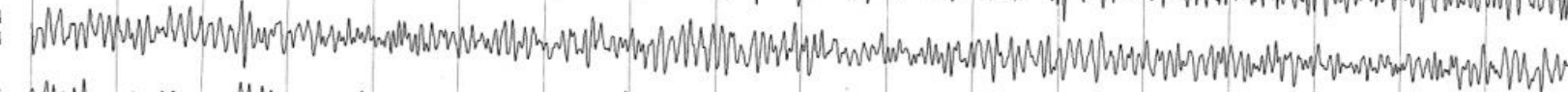

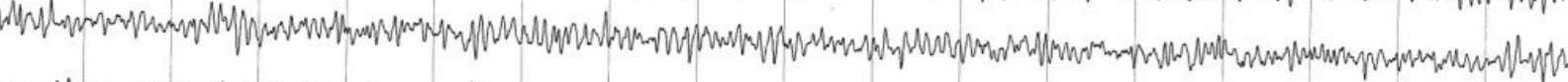

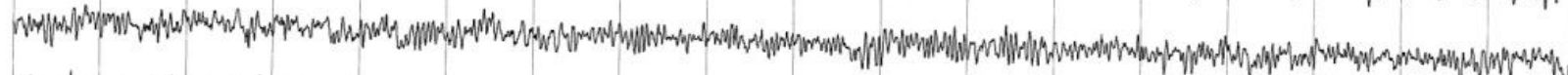

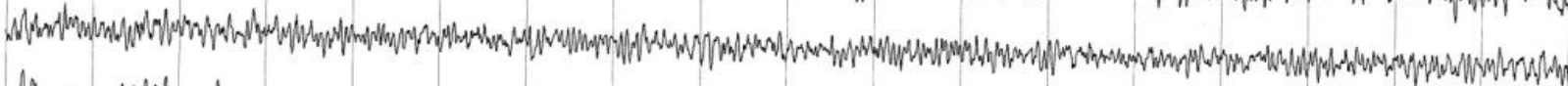

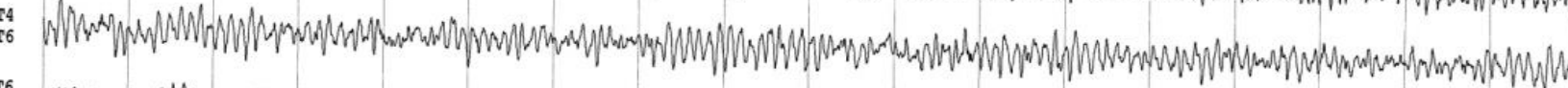

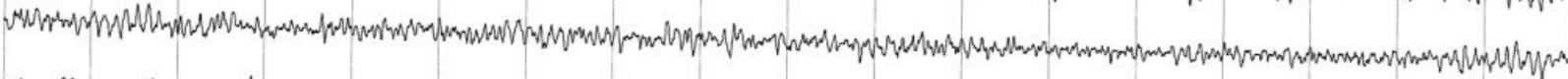

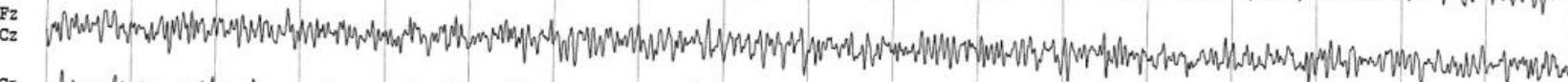

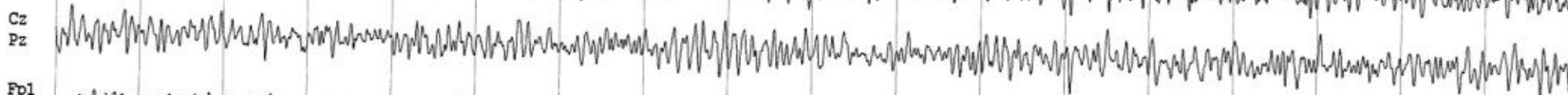

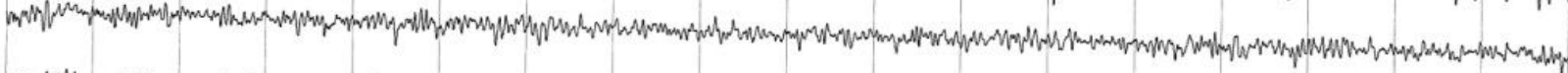

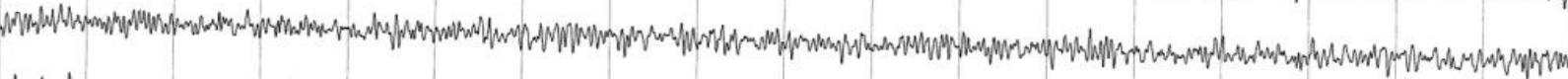

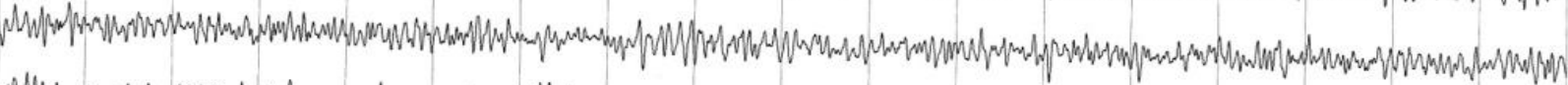

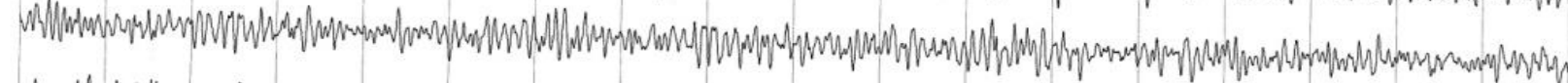

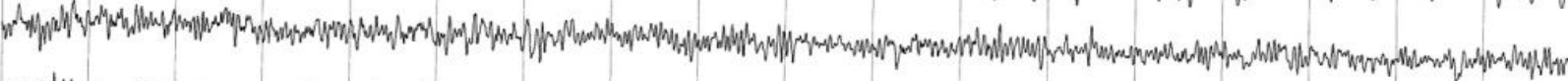

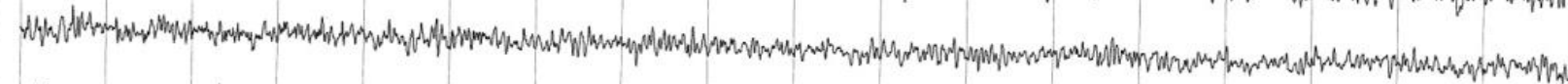

T3

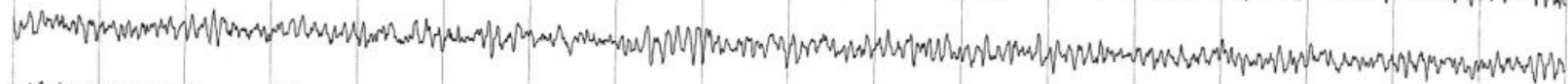

T5

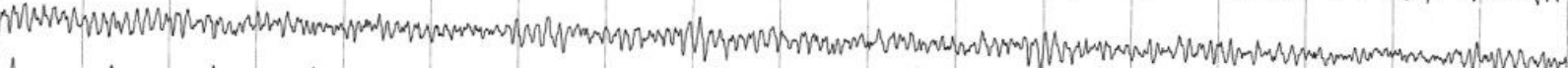

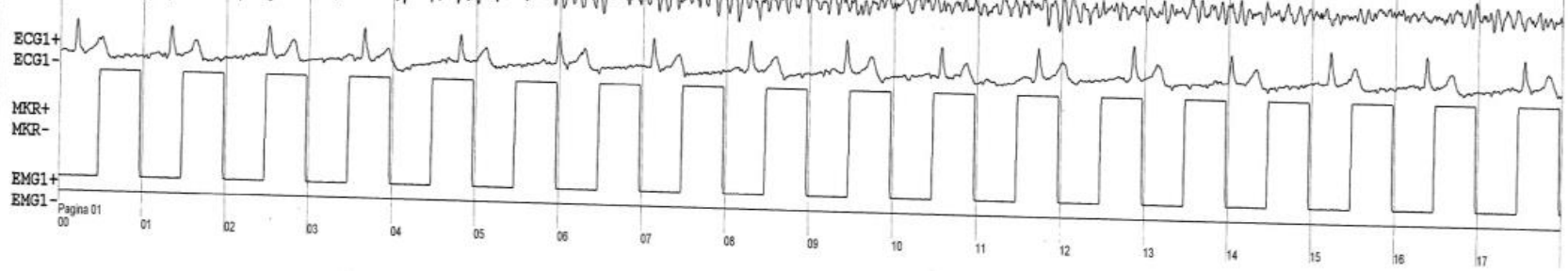

Figure 1: EEG monitoring before and after flumazenil injection.

A. Widespread low voltage $(10-15 \mu \mathrm{V})$, fast frequency activity $(13-16 \mathrm{~Hz})$, prominent over the frontal regions; B. 6 minutes after flumazenil injection. Reappearance of alpha-like frequency background rhythm $(8-12 \mathrm{~Hz})$. 
Citation: Fois C, Murgia B, Avallone R, Murrighile RM, Sechi GP (2015) Case Report: Idiopathic Recurrent Stupor, Still an Unsolved Issue. J Sleep Disor: Treat Care 4:4.

difficult to detect using conventional techniques such as immunoassay screening, GCMS and HPLC [5,6]. Thus, although this issue has been going on for the last two decades, still now, unrecognized toxic recurrent hypersomnia cannot be easily disclosed, in clinical practice, by routine available diagnostic tools. Therefore, a worldwide accepted and feasible proceeding method seems required for clarifying situations that might lead to important legal repercussion, potentially involving patient's safety and clinicians' responsibility and, as in our patient, to an ominous prognosis.

\section{References}

1. Rothstein JD, Guidotti A, Tinuper $P$, Cortelli $P$, Avoni $P$, et al. (1992) Endogenous benzodiazepine receptor ligands in idiopathic recurring stupor. Lancet 340: 1002-1004.
2. Plazzi G, Rye D, Vignatelli L, Riva R, Lugaresi E (2014) Idiopathic recurrent stupor: Munchausen by proxy and medical litigation. Sleep 37: 211.

3. Lugaresi E, Montagna P, Tinuper P, Plazzi G, Gallassi R, et al. (1998) Endozepine stupor. Recurring stupor linked to endozepine-4 accumulation. Brain 121: 127-133.

4. Cortelli P, Avallone R, Baraldi M, Zeneroli ML, Mandrioli J, et al. (2005) Endozepines in recurrent stupor. Sleep Med Rev 9: 477-487.

5. Granot R, Berkovic SF, Patterson S, Hopwood M, Drummer OH, et al. (2004) Endozepine stupor: disease or deception? A critical review. Sleep 27: $1597-$ 1599.

6. Granot R (2008) Encephalopathies related to exogenous or endogenous benzodiazepines. In: GianPietro Sechi, ed. Drug Related Encephalopathies. Nova Science Pub 363-410.

\section{Author Affiliations}

${ }^{1}$ Department of Clinical and Experimental Medicine, University of Sassari, Sassari, Italy

${ }^{2}$ Department of Life Sciences, University of Modena and Reggio Emilia, Modena, Italy

Submit your next manuscript and get advantages of SciTechnol submissions

* 50 Journals

* 21 Day rapid review process

* 1000 Editorial team

* 2 Million readers

* Publication immediately after acceptance

* Quality and quick editorial, review processing

Submit your next manuscript at • www.scitechnol.com/submission 